\title{
CLSI - EUCAST: COMPARISON OF ANTIBIOTIC-SUSCEPTIBILITY PROFILE OF ENTEROBACTERIACEAE OF ANIMAL ORIGIN ACCORDING TO THE STANDARDS
}

\author{
JOSMAN DANTAS PALMEIRA ${ }^{1,2 *}$ and HELENA FERREIRA ${ }^{1,2}$ \\ ${ }^{1}$ Microbiology - Biological Sciences Department, Faculty of Pharmacy, \\ University of Porto, Porto, Portugal \\ ${ }^{2}$ UCIBIO - Research Unit on Applied Molecular Biosciences, REQUIMTE, \\ Porto, Portugal
}

(Received: 7 March 2019; accepted: 28 May 2019)

Antimicrobial resistance is a relevant "One Health" issue that shows the need of comparison of isolates of different origins. In this way, guidelines for antimicrobial-resistance evaluation in animals are relevant in relation to human sources. This work aims to compare antimicrobial-resistance results of animal isolates considering CLSI and EUCAST guidelines. The comparison shows considerable differences in the results, which include antibiotics used as primary options in hospital infections. EUCAST showed the higher number of samples with resistance profiles than CLSI that indicates a more efficient scenario to the EUCAST to screen antibiotic-resistant bacteria. EUCAST was more consonant to the expected phenotype for ESBL producers, with higher index of resistance to oxyimino-beta-lactam antibiotics. The study shows that there are differences in the interpretative results using different guidelines, where the susceptibility test results concerning Enterobacteriaceae of animal origin are not always coincident in CLSI and EUCAST. EUCAST has proved to be the most reliable alternative for profile screening of antibiotic resistance, when compared to CLSI. We might say the same with respect to the ESBL-producing Enterobacteriaceae, in which EUCAST has proved to be more efficient about the demonstration of expected resistance profiles for the ESBL producers. These differences show that guideline selection might influence the therapeutic option. animal

Keywords: CLSI, EUCAST, Enterobacteriaceae, susceptibility testing,

*Corresponding author; E-mail: josmandantasp@gmail.com 


\section{Introduction}

Antibiotic resistance is a problem that has been going worse and worse in the past decades. Therefore, detection and monitoring of the resistant microorganisms and their mechanisms of resistance is of great importance. Antibiotic susceptibility is evaluated using directions from notable institutions that are recognized by the scientific community. The current leading institutions that supply these standards for antibiotic susceptibility evaluation are the Clinical and Laboratory Standards Institute (CLSI) from North America and the European Committee on Antimicrobial Susceptibility Testing (EUCAST) from Europe. The CLSI guidelines for Antibiotic Susceptibility Tests (ASTs) were mostly used in the world for many years, given its credibility (the Institute began as a National Committee - NCCLS almost 50 years ago) and were recognized in United States of America by Food and Drug Administration. Laboratories need to pay an annual signature to have access to the current version that is updated every year $[1,2]$. The EUCAST has been recognized at European level; it has been only 20 years and has emerged from the unification of several European reference institutions, which justifies its credibility and accessibility regarding the microbiology laboratories, once its content is available for free [3, 4].

At present, there are no reference standards for AST interpretation concerning microorganisms of animal origin in EUCAST. CLSI produces annually the CLSI VET, which establishes criteria for samples of animal origin, that is widely used in the United States [5]. However, for AST of bacteria of animal origin, whether it is for animal prescription or for scientific research purposes, in European reality, as for other countries, the human standard references of CLSI and EUCAST are the most used [6-11].

Several studies have analyzed the differences and similarities of the results obtained by the guidelines of both institutions regarding fungi and Gram-positive and Gram-negative bacteria, yet the data related to the comparative evaluation of results of Enterobacteriaceae from animal production are scarce and of great importance to the comprehension of the differences that might exist between these interpretations and their impact on resistance to antibiotics of the samples of animal origin, which much concerns human health when analyzed by the perspective of One Health [1, 12-18].

\section{Materials and Methods}

A range of 309 Enterobacteriaceae isolated from samples of healthy bovine feces, of the production environment (manure, ration, and water consumed by the 
animals), and of other animal products (milk), were submitted to AST evaluation by the agar diffusion method to 25 antimicrobials present on the interpretations by this method in CLSI [19] and EUCAST [20]. Nitrofurantoin and fosfomycin were analyzed only for Escherichia coli (266 strains). The samples from Brazilian animal productions were obtained in April 2014. The obtained results were classified as resistant, intermediate, or susceptible according to each institution. The data were inserted into the SSPS software version 25 (IBM, USA), in which the existent similarity between the obtained results by CLSI and EUCAST criteria was interpreted. In addition, the "kappa" coefficient from the relationship between results was analyzed to determine the relationship between both the results without the influence of the statistic random. It is interpreted between "perfect agreement" and "poor relationship."

\section{Results}

The 25 antimicrobials analyzed showed differences varying between $99.4 \%$ and $67.4 \%$. Those that showed high percentage of similarity were: sulfamethoxazole + trimethoprim (99.4\%), meropenem (98.4\%), amoxicillin (97.7\%), imipenem (97.7\%), netilmicin (97.6\%), and fosfomycin and amikacin $(96.7 \%)$. Studies performed using the 2016 version of CLSI and EUCAST also showed percentages of similarity above $95 \%$ for sulfamethoxazole + trimethoprim and meropenem [1]. Some antimicrobials showed low similarity values among the obtained results: ceftaroline $(67.4 \%)$, piperacillin + tazobactam $(73 \%)$, aztreonam (76.3\%), piperacillin (76.4\%), and ciprofloxacin (76.6\%). Table I shows similarity values and "kappa" coefficient analysis for the 25 antimicrobials analyzed.

The "kappa" coefficient analysis results show that the results of no antimicrobial are in perfect agreement once no "kappa" coefficient 1 was obtained. The best "kappa" coefficient results were classified as almost perfect regarding amoxicillin $-\mathrm{k}$ of $0.952(95 \% \mathrm{CI}=0.917,0.987)$, cefoxitin $-\mathrm{k}$ of $0.844(95 \% \mathrm{CI}=0.757$, $0.930)$, amikacin $-\mathrm{k}$ of $0.819(95 \% \mathrm{CI}=0.708,0.929)$, sulfamethoxazole + trimethoprim $-\mathrm{k}$ of $0.987(95 \% \mathrm{CI}=0.969,1.005)$, and chloramphenicol $-\mathrm{k}$ of $0.810(95 \% \mathrm{CI}=0.730,0.891)$. The classification "substantial" for the coefficient "kappa" was the result that showed the biggest antimicrobials number, as amoxicillin + clavulanic acid $-\mathrm{k}$ of $0.609(95 \% \mathrm{CI}=0.517,0.700)$, piperacillin $\mathrm{k}$ of $0.612(95 \% \mathrm{CI}=0.524,0.700)$, cefuroxime $-\mathrm{k}$ of $0.676(95 \% \mathrm{CI}=0.594$, $0.758)$, cefotaxime $\mathrm{k}$ of $0.717(95 \% \mathrm{CI}=0.632,0.803)$, cefepime $-\mathrm{k}$ of $0.741(95 \%$ $\mathrm{CI}=0.660,0.822)$, meropenem $-\mathrm{k}$ of $0.661(95 \% \mathrm{CI}=0.366,0.956)$, imipenem $\mathrm{k}$ of $0.749(95 \% \mathrm{CI}=0.565,0.933)$, doripenem $-\mathrm{k}$ of $0.717(95 \% \mathrm{CI}=0.594$, $0.840)$, levofloxacin $-\mathrm{k}$ of $0.672(95 \% \mathrm{CI}=0.579,0.765)$, gentamicin $-\mathrm{k}$ of 0.766 


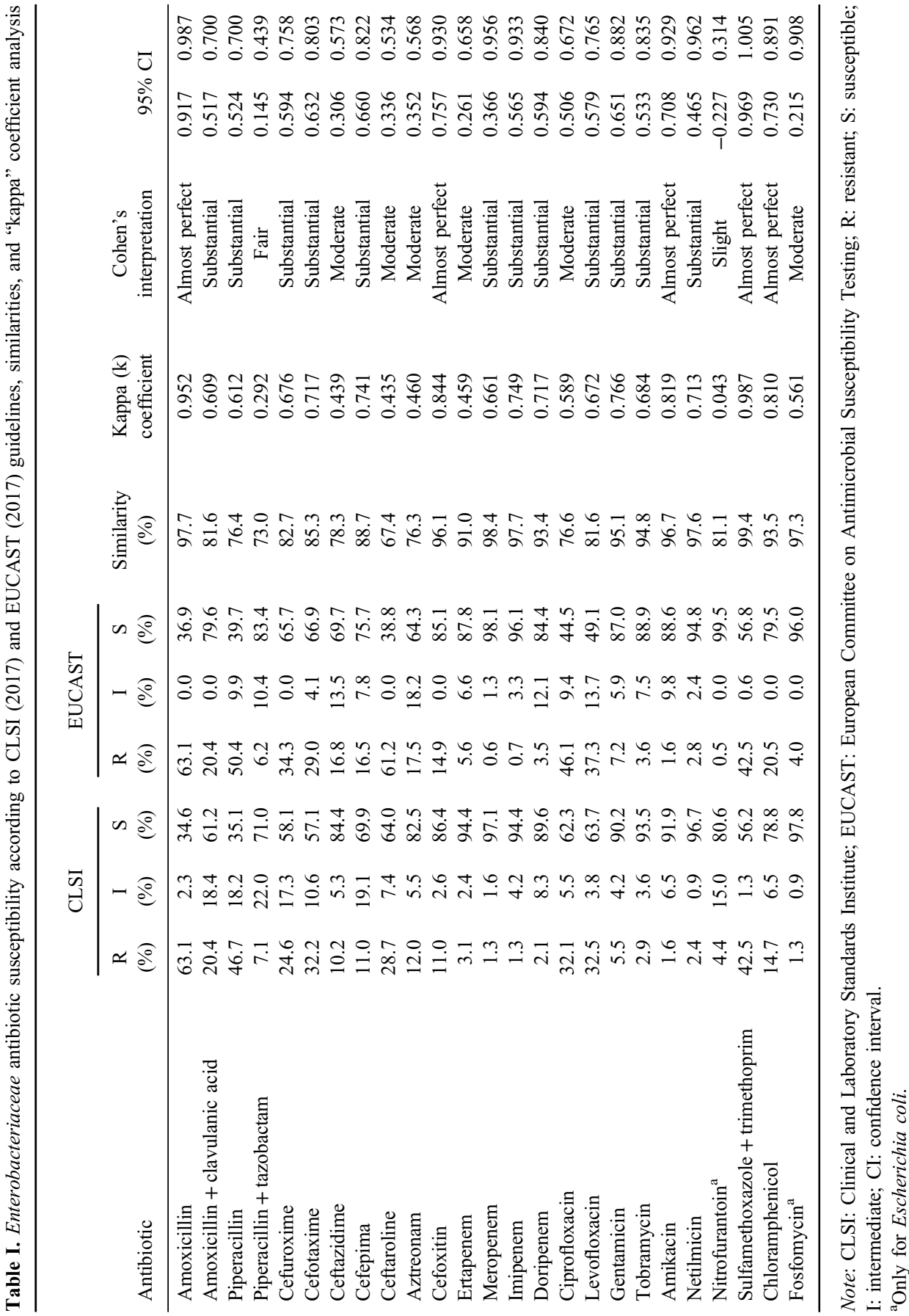


(95\% CI $=0.651,0.882)$, tobramycin $-\mathrm{k}$ of $0.684(95 \% \mathrm{CI}=0.533,0.835)$, and netilmicin $-\mathrm{k}$ of $0.713(95 \% \mathrm{CI}=0.465,0.962)$. The results classified as "moderate" are for ceftazidime - k of $0.439(95 \% \mathrm{CI}=0.306,0.573)$, ceftaroline $\mathrm{k}$ of $0.435(95 \% \mathrm{CI}=0.336,0.534)$, aztreonam $-\mathrm{k}$ of $0.460(95 \% \mathrm{CI}=0.352$, $0.568)$, ertapenem $-\mathrm{k}$ of $0.459(95 \% \mathrm{CI}=0.261,0.658)$, ciprofloxacin $-\mathrm{k}$ of $0.589(95 \% \mathrm{CI}=0.506,0.672)$, and fosfomycin $-\mathrm{k}$ of $0.561(95 \% \mathrm{CI}=0.215$, 0.908). Coefficient "kappa" results that showed the lowest classification, as "fair" and "slight," respectively, were piperacillin + tazobactam - k of $0.292(95 \% \mathrm{CI}=0.145,0.439)$ and nitrofurantoin $-\mathrm{k}$ of $0.043(95 \% \mathrm{CI}=-0.227$, $0.314)$.

When we compare the number of samples classified as resistant regarding both the standards, we discover a scenario in which few results are coincident. Four antibiotics (amoxicillin, amoxicillin + clavulanic acid, amikacin, and sulfamethoxazole + trimethoprim) showed similar results to the resistance profile concerning the susceptibility evaluation. In regard to five antibiotics (piperacillin + tazobactam, cefotaxime, meropenem, imipenem, and nitrofurantoin), the CLSI criteria showed more samples considered as resistant, when compared to EUCAST, with emphasis on nitrofurantoin (CLSI $-4.4 \%$ and EUCAST $0.5 \%$ ). The other 16 antibiotics (piperacillin, cefuroxime, ceftazidime, cefepime, ceftaroline, aztreonam, ertapenem, doripenem, ciprofloxacin, levofloxacin, gentamicin, tobramycin, netilmicin, chloramphenicol, and fosfomycin) showed higher number of resistant samples for EUCAST, presenting in some cases high discrepancy, as for ceftaroline (EUCAST - 61.2\% and CSLI - 28.7\%), ciprofloxacin (EUCAST - 46.1\% and CLSI - 32.1\%), and cefuroxime (EUCAST - 34.3\% and CLSI - 24.6\%). Figure 1 presents the resistance profile for all 25 antibiotics, concerning CLSI and EUCAST.

Among the 309 Enterobacteriaceae samples analyzed, 77 were extendedspectrum beta-lactamase producers (ESBL), and when we look at their susceptibility profile to third-, fourth-, and fifth-generation cephalosporins and to monobactams, it is observed that there is a higher number of samples classified as resistant (except for cefotaxime) in the EUCAST interpretation. This difference is very clear for ceftazidime (EUCAST - 29.1\% and CLSI - 12.7\%), cefepime (EUCAST - 53.2\% and CLSI - 33.8\%), and aztreonam (EUCAST - 29.9\% and CLSI $-15.6 \%$ ), once the number of isolates with resistance profiles is superior to the obtained by CLSI, and it might be the reason that masks the positive results of AST of possible ESBL producers that do not show the typical synergism. This resistance profile difference might show the hydrolytic strength for low-level betalactamases, when CSLI is utilized. Figure 2 illustrates the comparative profiles concerning the 77 ESBL-producing Enterobacteriaceae to the third-, fourth-, and fifth-generation cephalosporins and to monobactams. 


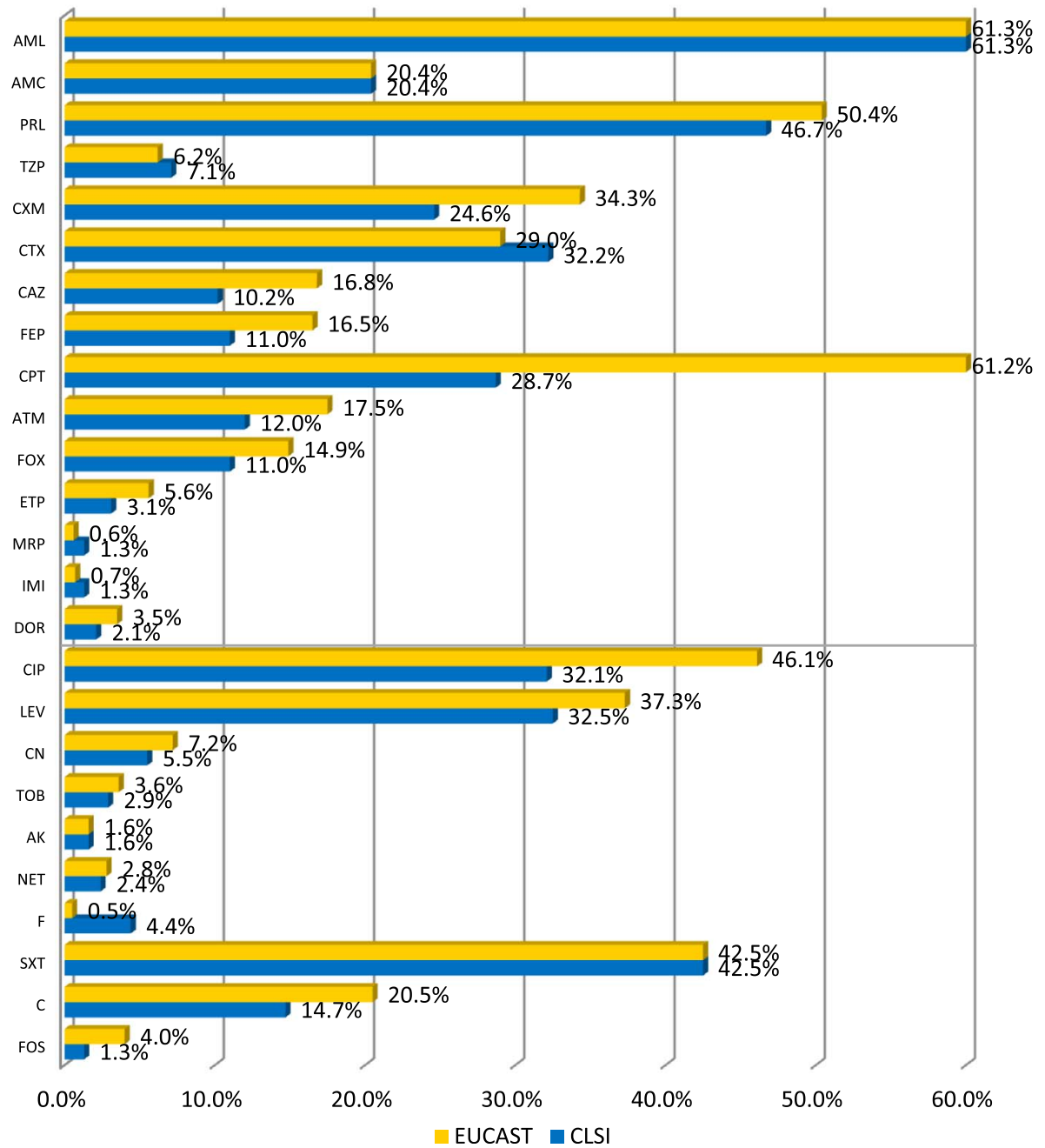

Figure 1. Antibiotic resistance profile of Enterobacteriaceae strains according to CLSI (2017) and EUCAST (2017) standards. AML: amoxicillin; AMC: amoxicillin + clavulanic acid; PRL: piperacillin; TZP: piperacillin + tazobactam; CXM: cefuroxime; CTX: cefotaxime; CAZ: ceftazidime; FEP: cefepime; CPT: ceftaroline; ATM: aztreonam; FOX: cefoxitin; ETP: ertapenem; MRP: meropenem; IMI: imipenem; DOR: doripenem; CIP: ciprofloxacin; LEV: levofloxacin; CN: gentamycin; TOB: tobramycin; AK: amikacin; NET: netilmicin; F: nitrofurantoin; SXT: sulfamethoxazole + trimetoprim; C: chloramphenicol; FOS: fosfomycin 


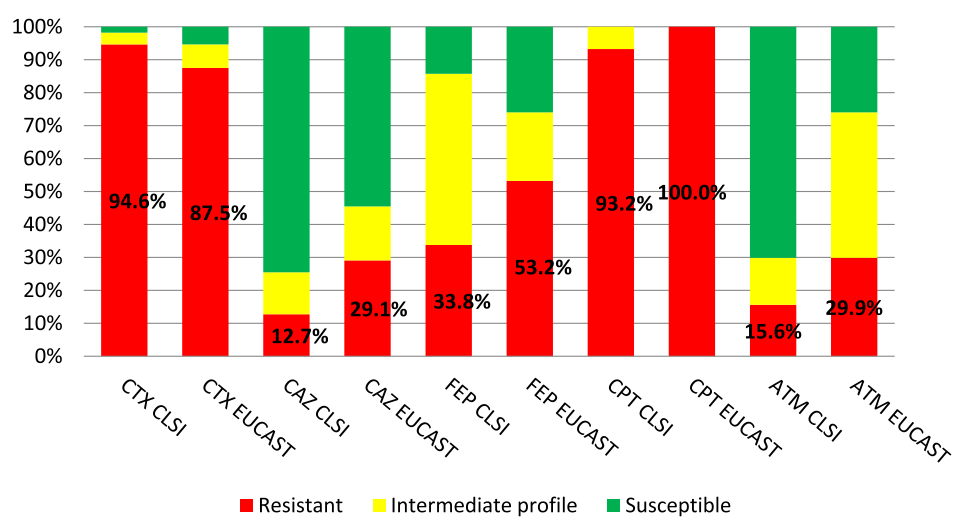

Figure 2. Susceptibility profile of ESBL-producing Enterobacteriaceae to third-, fourth-, and fifth-generation cephalosporins and to monobactams regarding CLSI (2017) and EUCAST (2017) standards. CTX: cefotaxime; CAZ: ceftazidime; FEP: cefepime; CPT: ceftaroline; ATM: aztreonam

\section{Discussion}

The results showed significant differences about the profile of resistance of Enterobacteriaceae of animal origin resistance when comparing CLSI and EUCAST standards. Some evaluated antibiotics have obtained high similarity according to Cohen's classification, such as amoxicillin, cefoxitin, amikacin, sulfamethoxazole + trimethoprim, and chloramphenicol, classified as "almost perfect." Also showing good similarity, we can find amoxicillin + clavulanic acid, piperacillin, cefuroxime, cefotaxime, cefepime, meropenem, imipenem, doripenem, levofloxacin, gentamicin, tobramycin, and netilmicin. However, six antibiotics showed similar results classified only as "moderate," such as ceftazidime, ceftaroline, aztreonam, ertapenem, ciprofloxacin, and fosfomycin, which are the antibiotics used as first options in hospital infections (ceftazidime and aztreonam) and as alternative for infections caused by ESBL producers (ciprofloxacin and fosfomycin). These differences show that the selection of the standard might influence the therapeutic option [21,22].

EUCAST showed the higher number of samples with resistance profiles more prominent than CLSI. This fact indicates a scenario in which EUCAST shows more efficient with respect to screening of the antibiotics resistance profile, revealing a higher susceptibility in animal samples, when compared to CLSI, given that for several antibiotics, EUCAST presented resistant samples, whereas in the CLSI evaluation, those would be classified as susceptible or intermediate.

With regard to the ESBL producers, the EUCAST results have proved to be more consonant to the expected phenotype for the Enterobacteriaceae that 
produces these enzymes, that is, higher index of resistance to third-, fourth-, and fifth-generation cephalosporins and to aztreonam. A study performed with human clinical samples has demonstrated that EUCAST is more efficient to the ESBL detection [23].

\section{Conclusions}

The similarity of the susceptibility test results concerning Enterobacteriaceae of animal origin is not always coincident in CLSI and EUCAST, due to different interpretations on the minimum inhibitory concentrations. Although EUCAST has proved to be the most reliable alternative for profile screening of resistance to antibiotics, once it has shown more efficient concerning detection of resistance profiles by the agar diffusion method, when compared to CLSI. We might say the same with respect to the ESBL-producing Enterobacteriaceae, in which EUCAST has proved to be more efficient about the demonstration of expected resistance profiles for the producers of these enzymes.

\section{Acknowledgements}

This work was supported by a scholarship of Coordination for the Improvement of Higher Education Personnel (CAPES).

\section{Conflict of Interest}

The authors declare no competing interests.

\section{References}

1. Kassim, A., Omuse, G., Premji, Z., Revathi, G.: Comparison of Clinical Laboratory Standards Institute and European Committee on Antimicrobial Susceptibility Testing guidelines for the interpretation of antibiotic susceptibility at a University teaching hospital in Nairobi, Kenya: A cross-sectional study. Ann Clin Microbiol Antimicrob 15, 21 (2016).

2. Clinical and Laboratory Standards Institute (CLSI): CLSI Standards: Guidelines for Health Care Excellence. CLSI, Wayne, PA, 2018.

3. Kahlmeter, G.: The 2014 Garrod Lecture: EUCAST - Are we heading towards international agreement? J Antimicrob Chemother 70, 2427-2439 (2015).

4. European Committee on Antimicrobial Susceptibility Testing (EUCAST): Clinical Breakpoints. EUCAST, 2018. 
5. Dargatz, D. A., Erdman, M. M., Harris, B.: A survey of methods used for antimicrobial susceptibility testing in veterinary diagnostic laboratories in the United States. J Vet Diagn Invest 29, 669-675 (2017).

6. Poirel, L., Nordmann, P., Ducroz, S., Boulouis, H.-J., Arné, P., Millemann, Y.: Extendedspectrum $\beta$-lactamase CTX-M-15-producing Klebsiella pneumoniae of sequence type ST274 in companion animals. Antimicrob Agents Chemother 57, 2372-2375 (2013).

7. Su, Y., Yu, C.-Y., Tsai, Y., Wang, S.-H., Lee, C., Chu, C.: Fluoroquinolone-resistant and extended-spectrum $\beta$-lactamase-producing Escherichia coli from the milk of cows with clinical mastitis in Southern Taiwan. J Microbiol Immunol Infect 49, 892-901 (2016).

8. Webb, H. E., Bugarel, M., den Bakker, H. C., Nightingale, K. K., Granier, S. A., Scott, H. M., Loneragan, G. H.: Carbapenem-resistant bacteria recovered from faeces of dairy cattle in the high plains region of the USA. PLoS One 11, e0147363 (2016).

9. Aslantaş, Ö., Elmacıkoğlu, S., Yilmaz, E. Ş.: Prevalence and characterization of ESBL- and AmpC-producing Escherichia coli from cattle. Kafkas Universitesi Veteriner Fakultesi Dergisi 23, 63-67 (2017).

10. Barzan, M., Gharibi, D., Ghorbanpoor, M., Haji Hajikolaei, M., Pourmehdi-Boroujeni, M.: Phylogenetic grouping and phenotypic detection of extended-spectrum $\beta$-lactamases among Escherichia coli from calves and dairy cows in Khuzestan, Iran. Int J Enteric Pathog 5, 24-29 (2017).

11. Jouy, E., Haenni, M., Le Devendec, L., Le Roux, A., Châtre, P., Madec, J.-Y., Kempf, I.: Improvement in routine detection of colistin resistance in $E$. coli isolated in veterinary diagnostic laboratories. J Microbiol Methods 132, 125-127 (2017).

12. Chryssanthou, E., Cuenca-Estrella, M.: Comparison of the EUCAST-AFST broth dilution method with the CLSI reference broth dilution method (M38-A) for susceptibility testing of posaconazole and voriconazole against Aspergillus spp. Clin Microbiol Infect 12, 901-904 (2006).

13. Vading, M., Samuelsen, Ø., Haldorsen, B., Sundsfjord, A. S., Giske, C. G.: Comparison of disk diffusion, Etest and VITEK2 for detection of carbapenemase-producing Klebsiella pneumoniae with the EUCAST and CLSI breakpoint systems. Clin Microbiol Infect 17, 668-674 (2011).

14. Asín, E., Isla, A., Canut, A., Rodríguez Gascón, A.: Comparison of antimicrobial pharmacokinetic/pharmacodynamic breakpoints with EUCAST and CLSI clinical breakpoints for Gram-positive bacteria. Int J Antimicrob Agents 40, 313-322 (2012).

15. Marchese, A., Esposito, S., Barbieri, R., Bassetti, M., Debbia, E.: Does the adoption of EUCAST susceptibility breakpoints affect the selection of antimicrobials to treat acute community-acquired respiratory tract infections? BMC Infect Dis 12, 181 (2012).

16. Wolfensberger, A., Sax, H., Weber, R., Zbinden, R., Kuster, S. P., Hombach, M.: Change of Antibiotic Susceptibility Testing Guidelines from CLSI to EUCAST: Influence on Cumulative Hospital Antibiograms. PLoS One 8, e79130 (2013).

17. Pfaller, M. A., Castanheira, M., Messer, S. A., Rhomberg, P. R., Jones, R. N.: Comparison of EUCAST and CLSI broth microdilution methods for the susceptibility testing of 10 systemically active antifungal agents when tested against Candida spp. Diagn Microbiol Infect Dis 79, 198-204 (2014).

18. Arendrup, M. C., Prakash, A., Meletiadis, J., Sharma, C., Chowdhary, A.: Comparison of EUCAST and CLSI reference microdilution MICs of eight antifungal compounds for 
Candida auris and associated tentative epidemiological cutoff values. Antimicrob Agents Chemother 61, e00485-17 (2017).

19. Clinical and Laboratory Standards Institute (CLSI): Performance Standards for Antimicrobial Susceptibility Testing, 27th ed. CLSI supplement M100. Clinical and Laboratory Standards Institute, Wayne, PA, 2017.

20. European Committee on Antimicrobial Susceptibility Testing (EUCAST): Breakpoint Tables for Interpretation of MICs and Zone Diameters. Version 7.1. EUCAST, 2017.

21. Lo, C.-L., Lee, C.-C., Li, C.-W., Li, M.-C., Hsueh, P.-R., Lee, N.-Y., Ko, W.-C.: Fluoroquinolone therapy for bloodstream infections caused by extended-spectrum beta-lactamase-producing Escherichia coli and Klebsiella pneumoniae. J Microbiol Immunol Infect 50, 355-361 (2017).

22. Yeganeh-Sefidan, F., Ghotaslou, R., Akhi, M. T., Sadeghi, M. R., Mohammadzadeh-Asl, Y., Bannazadeh Baghi, H.: Fosfomycin, interesting alternative drug for treatment of urinary tract infections created by multiple drug resistant and extended spectrum $\beta$-lactamase producing strains. Iran J Microbiol 8, 125-131 (2016).

23. Polsfuss, S., Bloemberg, G. V., Giger, J., Meyer, V., Hombach, M.: Comparison of European Committee on Antimicrobial Susceptibility Testing (EUCAST) and CLSI screening parameters for the detection of extended-spectrum $\beta$-lactamase production in clinical Enterobacteriaceae isolates. J Antimicrob Chemother 67, 159-166 (2012). 